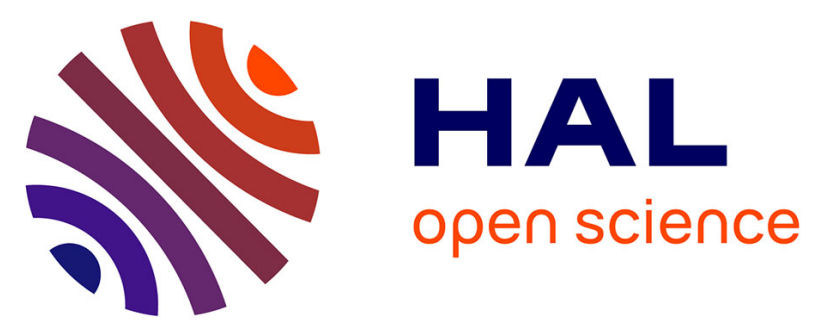

\title{
Femtosecond spin-state photo-switching dynamics in an FeIII spin crossover solid accompanied by coherent structural vibrations
}

\author{
Roman Bertoni, Maciej Lorenc, Jerome Laisney, Antoine Tissot, Alain \\ Moréac, Samir F. Matar, Marie-Laure Boillot, Eric Collet
}

\section{To cite this version:}

Roman Bertoni, Maciej Lorenc, Jerome Laisney, Antoine Tissot, Alain Moréac, et al.. Femtosecond spin-state photo-switching dynamics in an FeIII spin crossover solid accompanied by coherent structural vibrations. Journal of Materials Chemistry C, 2015, 3 (30), pp.7792-7801. 10.1039/C5TC00854A . hal-01152947

\section{HAL Id: hal-01152947 \\ https://hal.science/hal-01152947}

Submitted on 20 May 2015

HAL is a multi-disciplinary open access archive for the deposit and dissemination of scientific research documents, whether they are published or not. The documents may come from teaching and research institutions in France or abroad, or from public or private research centers.
L'archive ouverte pluridisciplinaire HAL, est destinée au dépôt et à la diffusion de documents scientifiques de niveau recherche, publiés ou non, émanant des établissements d'enseignement et de recherche français ou étrangers, des laboratoires publics ou privés. 


\title{
Femtosecond spin-state photo-switching dynamics in an Fe"' spin crossover solid accompanied by coherent structural vibrations
}

\author{
R. Bertoni, ${ }^{a}$ M. Lorenc, ${ }^{a}$ J. Laisney ${ }^{b}$, A. Tissot $^{b,+},{ }^{+}$A. Moréac ${ }^{a}$, S. F. Matar ${ }^{c}$, M.-L. Boillot ${ }^{b}$ and \\ E. Collet ${ }^{\mathrm{a}, *}$
}

\begin{abstract}
We investigate light-induced excited spin-state trapping (LIESST) dynamics of an Fe"' spin-crossover material from low $(S=1 / 2)$ to high $(S=5 / 2)$ spin states. Our results show that this process occurs only at the molecular level as evidenced by the linear dependence of the fraction of photo-switched molecules with the excitation density as well as with the initial fraction of low spin molecules. The inter-system crossing from photoexcited LS ( $S=1 / 2)$ to HS ( $S=5 / 2)$ occurs within $\approx 200$ fs and is accompanied by coherent non-equilibrium vibrational relaxation in the photo-induced HS state. These results reveal similar dynamical features to those already reported for LIESST in Fe" systems. The activation of coherent molecular vibrations is essential for reaching rapidly the HS potential on the timescale of molecular motions, whereas their fast damping allows an efficient trapping in the HS potential. The observed coherent oscillations are attributed to photoinduced molecules in the HS states, as supported by Raman spectroscopy at thermal equilibrium, and DFT analyses of molecular vibrations and TD-DFT calculations of optical absorption.
\end{abstract}

\section{Introduction}

In molecular science, light is known to be an efficient trigger for driving chemical reactions, breaking bonds or changing electronic molecular states ${ }^{1}$. Reaction coordinates connecting initial, photoexcited, and final photoinduced states are not simple to describe. Furthermore, the pathway followed on the potential energy surface is complex and numerous studies investigated how fast such processes are and how interconversion of molecular states proceeds. In the solid state too, light can switch molecules between different electronic states. Charge-transfer processes induced by light can lead to photo-induced phase transition from insulating to metallic ${ }^{2}$ or from neutral to ionic ${ }^{3}$ phases for example. Spin-crossover (SCO) materials are bistable molecular systems able to switch between low spin (LS) and high spin (HS) states under the effect of different control parameters such as temperature or pressure ${ }^{4}$. They are also prototype photoactive systems as they can be interconverted through the light-induced excited spin-state trapping (LIESST) process from LS to HS state or conversely from HS to LS state (reverse-LIESST). LIESST in crystals was discovered more than thirty years ago ${ }^{5}$ by

\footnotetext{
a. Institut de Physique de Rennes, Université de Rennes1, UMR UR1-CNRS 6251 F-35000 Rennes, France.

${ }^{b}$ Institut de Chimie Moléculaire et des Matériaux d'Orsay, Université Paris-Sud, UMR-CNRS 8182, Orsay, France.

c. CNRS, Université de Bordeaux, ICMCB, 87 avenue du Dr. A. Schweitzer, Pessac, 33608 France.

* Corresponding author Email address: eric.collet@univ-rennes1.fr

+ Present address: Institut Lavoisier de Versailles, UMR CNRS 8180, Université de Versailles Saint-Quentin, 78035 Versailles cedex, France.

Electronic Supplementary Information (ESI) available: Videos of the molecular vibrational modes calculated at $56 \mathrm{~cm}^{-1}, 33 \mathrm{~cm}^{-1}$ and $88 \mathrm{~cm}^{-1}$.
}

irradiating SCO solids with $\mathrm{cw}$ light at low temperature complete conversion of molecular states can be generated at the macroscopic scale of the material and the photo-induced state can be metastable up to a critical temperature referred to as $\left.\mathrm{T}^{(\mathrm{LIESST}}\right)^{6}$. In crystals, molecules may respond cooperatively to light excitation ${ }^{2,3}$. In addition, light can control order between the individual molecules of the materials and generate new phases which cannot be reached at thermal equilibrium ${ }^{7}$. This underlines the ability of molecular-based materials to show response to light different from the response of the individual constituting molecules. There are also other examples in the literature of molecular materials showing cooperative response to light excitation, for which a single photon transforms several molecules and the response of the molecular material is more than the sum of individual molecular events ${ }^{2,3}$. Spin-crossover has become a topical research area over the last three decades, with many research groups contributing to its advancement ${ }^{4}$. The very high efficiency and speed of the photo-induced spin state switching between two states that completely differ in physical properties (color, volume, magnetic susceptibility) represent a conceptual challenge ${ }^{8}$. For Fe" systems the role of ligand-field states was deeply investigated ${ }^{9}$. The study of reverse-LIESST revealed a mechanism involving two main inter-system crossing (ISC) and therefore true intermediate electronic states (such as $\mathrm{S}=1$ ) between the photoexcited HS $(\mathrm{S}=2)$ and the photoinduced LS $(\mathrm{S}=0)$ state, which is reached within 40 ps. But for LIESST, the irradiation into metal-ligand charge transfer (MLCT) or $d-d$ bands of $S=0$ species promotes the system to the $S=2$ state within $\approx 160 \mathrm{fs}$. This timescale was determined with ultrafast optical spectroscopy, a powerful technique for investigating the photo-physical dynamics of 
SCO compounds ${ }^{10}$. Complementary ultrafast X-ray absorption spectroscopy studies revealed the structural dynamics related to ligand-cage dilation, which leads to the structural trapping of the newly formed HS electronic state ${ }^{11}$. On the pathway from MLCT to HS states, other not structurally relaxed electronic states serve as intermediates. Indeed, these states are populated on a timescale shorter than an oscillation period in the potential ${ }^{12}$.

For better understanding this process, it seems necessary to track the electronic and structural pathway followed by the system with the time resolution of elementary electronic and molecular events. Recent theoretical works suggested that such speed and efficiency of LIESST results from the instantaneous activation of molecular phonons after photoexcitation ${ }^{13}$. This is due to the important displacive nature of the inter-system crossing, which moves the system rapidly into the HS potential with $a \approx 0.2 \AA$ elongation of Fe- $\mathrm{N}$ bonds. The experimental proof of such a conjecture was reported recently for an $\mathrm{Fe}^{\prime \prime}$ system ${ }^{14}$. This study revealed that the structural molecular reorganization, trapping the photoinduced electronic state, occurs in a two-step sequence: the molecule elongates first (within 170 femtosecond) and bends afterwards, via a coherent vibrational energy transfer between these two main structural modes.

There are very few $\mathrm{Fe}^{\mathrm{III}}$ materials showing efficient or longlived LIESST ${ }^{15}$ but we evidenced in several SCO archetypes ${ }^{16,17}$ by time-resolved spectroscopy that transient $\mathrm{Fe}^{\text {III }} \mathrm{HS}$ state can be generated. This is also the case of the $\mathrm{Fe}^{\text {III }} \mathrm{SCO}$ [ $\mathrm{Fe}(3-\mathrm{MeO}-$ SalEen) $\mathrm{PF}_{6}$ material studied here in the form of nanocrystals (average size, $\left.900(230) \times 285(65) \times 64(18) \mathrm{nm}^{3}\right)^{8 a}$. Here we show that the spin-state photo-switching dynamics in this $\mathrm{Fe}^{\prime \prime \prime}$ material occurs locally and that the structural trapping during LIESST is accompanied by coherent molecular dynamics.

\section{Results and discussion}

\section{Thermal spin conversion.}

The $\mathrm{Fe}^{\prime \prime \prime}$ complex [Fe(3-MeO-SalEen) $\left.{ }_{2}\right] \mathrm{PF}_{6}$ was originally synthesized by Hendrickson ${ }^{18}$. H-3-MeO-SalEen is the condensation product of 3-methoxy-substituted salicylaldehyde and $\mathrm{N}$-ethyl-ethylenediamine. This Fe"I system can switch under the effect of external parameter, such as temperature, between LS and HS states. Since the HOMO and LUMO orbitals have a strong $d$ character, the two electronic states correspond to:

- a low spin one (LS) with $S=1 / 2$ with an electronic configuration $t_{2 g}^{5} e_{g}{ }^{0}$ in the octahedral-like description. More rigorously, in the symmetry of the complex the electronic configuration is $d_{\pi}^{5} d_{\sigma}{ }^{0}$.

- a high spin one (HS) with $S=5 / 2$ corresponding to the electronic configuration $t_{2 g}{ }^{3} e_{g}^{2}$ (or $\left.d_{\pi}{ }^{3} d_{\sigma}^{2}\right)$.

In the form of single crystals, this compound shows a firstorder phase transition from LS to HS phases at thermal equilibrium ${ }^{18,19}$. Nano-crystals of this material dispersed in
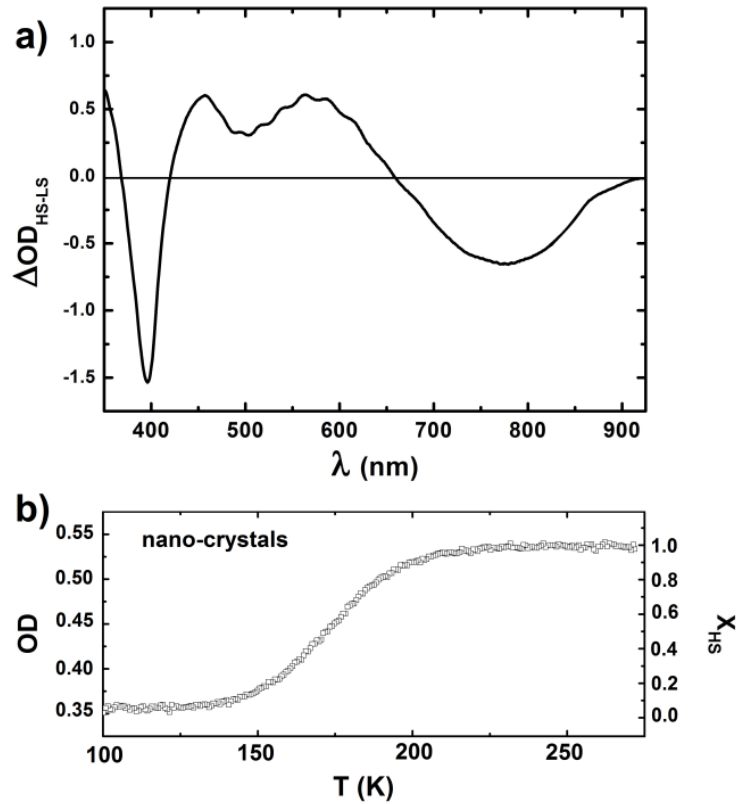

Fig.1 Difference of optical density in the (350-925 nm) spectral window between HS state $(296 \mathrm{~K})$ and LS state (16 K) measured on the nanocrystals sample (a). Photo-excitation is performed at $850 \mathrm{~nm}$ in the tail of the LS absorption band. OD at $550 \mathrm{~nm}$ (left axis) of the nano-crystal assembly during the crossover scaled to $X_{H S}$ (right axis) (b).

polymeric matrix lose the $1^{\text {st }}$ order transition and show a more gradual spin conversion ${ }^{16}$, as a result of both the pressure effects and anchoring processes (physisorption, capillarity) due to the polymers ${ }^{20}$. It was recently demonstrated by timeresolved studies that these $\mathrm{Fe}^{\mathrm{III}}$ nanocrystals exhibit photoswitching toward a transient HS state by photo-excitation of the LS state ${ }^{8 a}$. Here we present new results and detailed analysis of the spin state photo-switching dynamics by means of femtosecond pump-probe optical spectroscopy. It is easier to investigate an assembly of nano-crystals dispersed in a PVP polymer film than single crystals: the low optical density of the nano-crystals allows to perform a detailed study over a broad spectral range from UV to IR. Single crystals restrict the range of wavelengths for spectroscopic probing due to the higher optical density (OD) ${ }^{8 a, 16 a}$.

Fig. 1 shows the variation of optical density $\triangle O D$ measured on the films of nano-crystals, between pure LS state $(16 \mathrm{~K})$ and pure HS state $(296 \mathrm{~K})$. It reveals phenolate-to-iron chargetransfer bands (CT) in the near-infrared (NIR) - visible (VIS) with distinct features for LS and HS states. With respect to that of LS state, the OD of the HS state is higher in VIS and lower in NIR parts of the spectrum, with a well-defined isosbestic point around $650 \mathrm{~nm}$. The thermal fraction of HS molecules $X_{H S}(T)$ is obtained by scaling the change of optical density $O D(T)$ at a given temperature $T$ to the change of optical density between LS $\left(O D_{L S}\right)$ and $\mathrm{HS}\left(O D_{H S}\right)$ states:

$$
X_{H S}(T)=\frac{O D(T)-O D_{L S}}{\Delta O D_{H S-L S}}
$$

The thermal behavior of $X_{H S}(T)$ obtained in this way is displayed in Fig. $3(\mathrm{~b})$ and indicates a gradual conversion spanning from $140 \mathrm{~K}$ to $220 \mathrm{~K}$, in agreement with magnetic measurements ${ }^{16}$. 


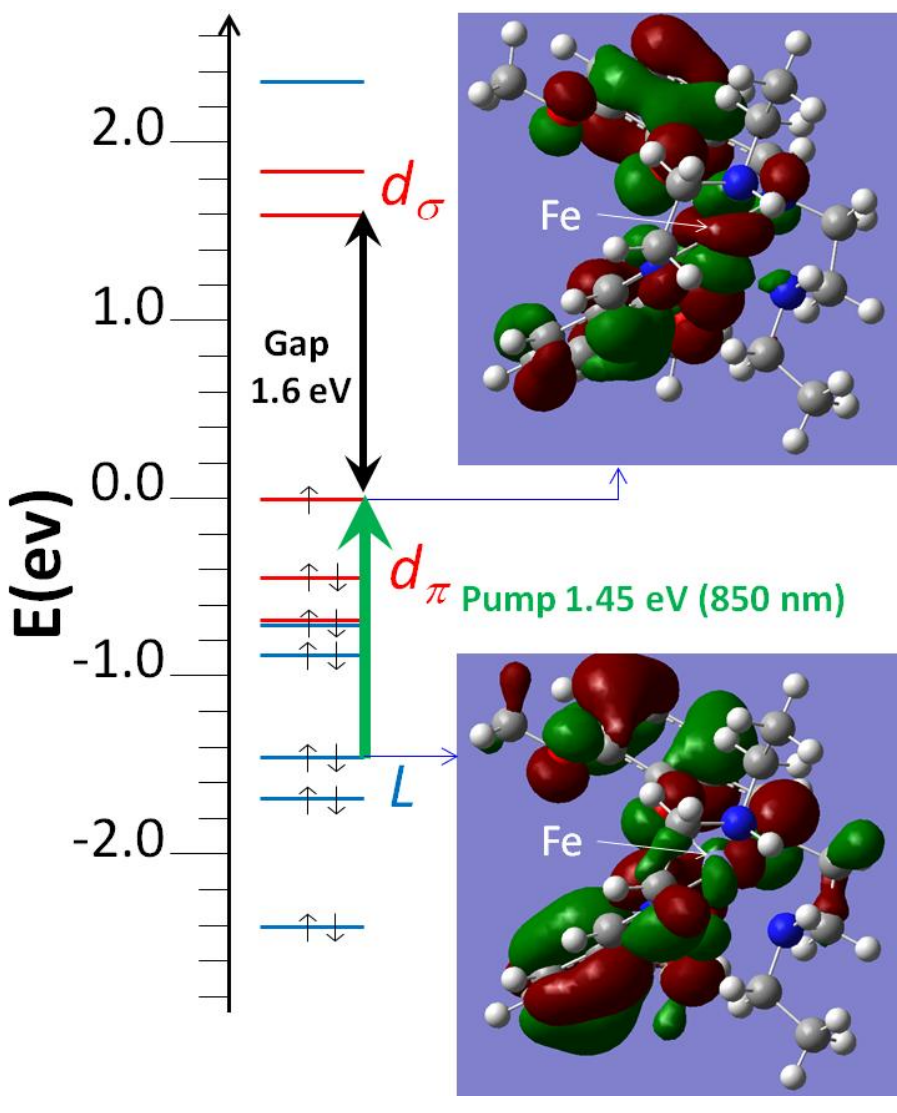

Fig. 2. LS schematic energy diagram and frontier molecular orbitals obtained from DFT calculations. Red: $d_{\sigma}$ and $d_{\pi}$ metal-like orbitals, blue : ligand-like orbitals. The HOMO-LUMO gap (1.6 eV) is larger than the pump excitation at $1.45 \mathrm{eV}(850 \mathrm{~nm})$. With respect to the HOMO (strong weight on a Fe d orbital), a few doubly occupied molecular orbitals (centered on phenol groups) falls within the energy range corresponding to the photo-excitation.

In the following, we exploit these spectroscopic fingerprints, to study the ultrafast spin-state photo-switching and the resulting energy dissipation into the material.

\section{Inter-System Crossing and vibrational cooling}

The spin-state photo-switching is known to proceed via transient intermediate states, as the direct low energy excitation from LS to HS state is forbidden by spin parity. In the case of Fe" systems, the photo-switching at the molecular level results from the promotion by light of an electron from the metal center to the ligand orbitals (MLCT excited state). For this $\mathrm{Fe}^{\mathrm{III}}\left[\mathrm{Fe}(3-\mathrm{MeO}-\mathrm{SalEen})_{2}\right] \mathrm{PF}_{6}$ system, we performed DFT calculations with Gaussian 09 package to establish the molecular orbital energy diagram. Fig. 2 shows that in the LS state the highest occupied molecular orbital (HOMO) and lowest unoccupied molecular orbitals (LUMO), both of Fe d predominant character, are separated by a $1.6 \mathrm{eV}$ energy gap. We found that the low energy excitation at $850 \mathrm{~nm}(1.45 \mathrm{eV})$ therefore may promote an electron from a few symmetryadapted and doubly-occupied molecular orbitals (centered on the phenol group, noted $L$ ) to the HOMO with a strong weight on the $d$ orbital of the metal center $(M)$. This LMCT state corresponds then to a $L^{1} d_{\pi}^{6} d_{\sigma}{ }^{0}$ electronic configurations.

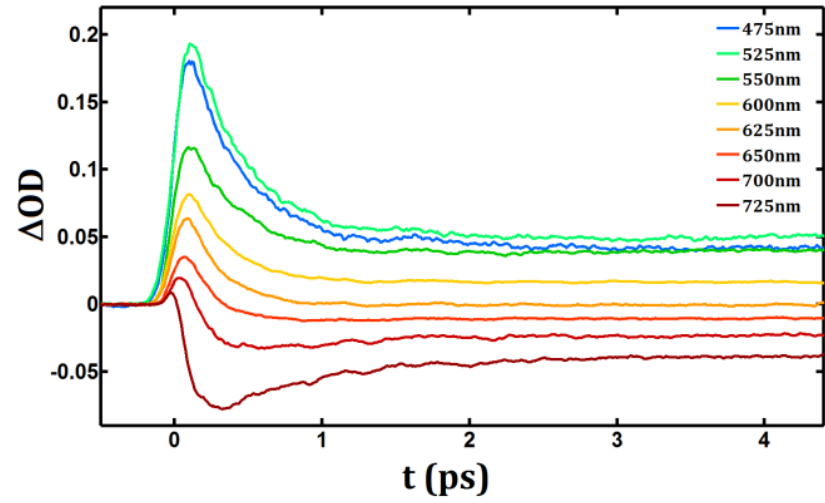

Fig. 3. Change of optical density at different probe wavelengths after $\mathrm{fs}$ excitation at $850 \mathrm{~nm}$ measured on [Fe"'(3-MeO-SalEen) ${ }_{2} \mathrm{PF}_{6}$ nanocrystals. Measurements are performed at $100 \mathrm{~K}$ in pure LS state.

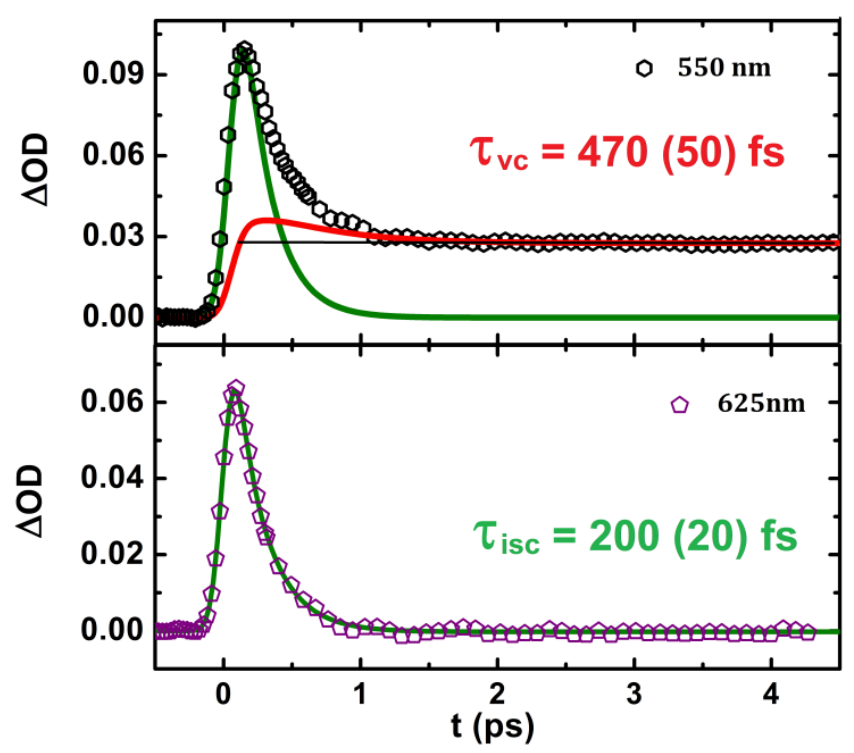

Fig. 4. Deconvolution of raw data into the relaxation kinetic of the INT electronic states $\left(\tau_{\text {isc, }}\right.$ green line) and the population kinetic of the HS state taking into account vibrational cooling ( $\tau_{\mathrm{vc}}$, red line). Only $1 / 8$ of the experimental data are shown for clarity.

By using femtosecond optical spectroscopy (see experimental section), it is possible to study the photo-switching dynamics since the different molecular states have peculiar optical spectra (Fig. 1). Selected time traces of transient OD measurements performed at different probing wavelengths are plotted in Fig. 3-5. The OD increase in the VIS part and bleaching in the NIR part (at the ps timescale), are characteristic of the formation of the HS state, as observed during the thermal LS to HS conversion (Fig. 1) and previously reported on similar samples ${ }^{8 a, 16}$.

Around time zero, the OD spectrum differs significantly from those of the LS ground state and of the HS photoinduced state, in terms of intensities or spectral range, where OD increases or decreases. This is the optical fingerprint of the LMCT state. The kinetic evolution of the optical density around the isosbestic point at $625 \mathrm{~nm}$, where the OD of LS and photoinduced HS states are the same ${ }^{8 a}$, allows to selectively follow the dynamics of this intermediate (Franck-Condon) state. 


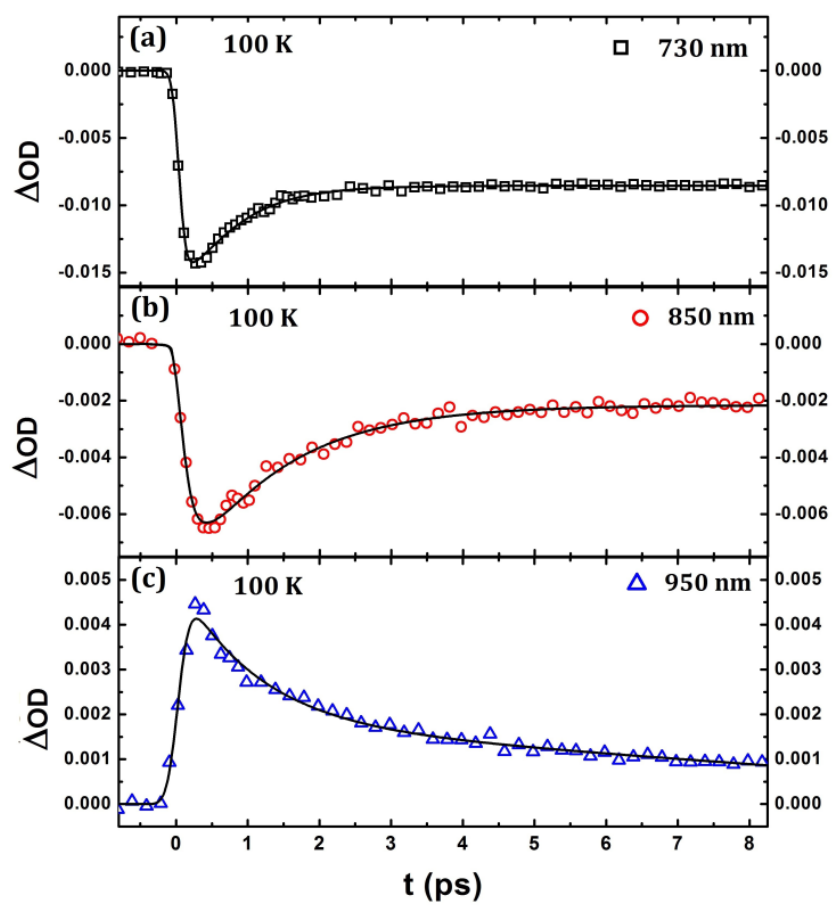

Fig. 5. Fit of the $\triangle O D$ traces at $730,850 \& 950 \mathrm{~nm}$ with bi-exponential functions describing ISC and vibrational cooling.

The OD kinetic trace at $625 \mathrm{~nm}$ shows at first a rapid rise (limited by our time resolution) corresponding to the generation of the LMCT state, which then decays towards the final HS state. Rigorously speaking, the dynamics may involve a sequence of transient states between LS and HS, as already reported in the case of $\mathrm{Fe}^{\prime \prime}$ systems. But since we do not observe clear fingerprints of different intermediate states, we consider only a lump intermediate state (INT) including the LS LMCT state $\left(L^{1} d_{\pi}{ }^{6} d_{\sigma}{ }^{0}\right.$ electronic configuration) and other possible intermediates, such as those resulting from $L^{1} d_{\pi}{ }^{5} d_{\sigma}{ }^{1}$ or $L^{1} d_{\pi}^{4} d_{\sigma}^{2}$ electronic configurations.

The inter-system crossing (ISC) sequence from LMCT to HS is well described at $625 \mathrm{~nm}$ by a single exponential decay. The fit shown in Fig. 4 gives $\tau_{I S C}=200 \pm 20$ fs. For other measurements with probe wavelengths away from the isosbestic point, the single exponential model fails to reproduce the observed dynamics and slower components are observed on the ps time-scale, as shown at $550 \mathrm{~nm}$ for example. This is especially true in the IR region $(950 \mathrm{~nm}$, Fig. $5)$, more sensitive to vibrational cooling since lower energetic levels are probed. Thus we used a two-exponential decay model for fitting both dynamics, the first time constant describing the ISC being fixed to $\tau_{I S C}=200 \mathrm{fs}$. Depending on the probe wavelength the vibrational cooling time constant $\tau_{v c}$ varies from $470(50)$ fs at $550 \mathrm{~nm}$ and up to $1.6(0.2) \mathrm{ps}$ at 950 $\mathrm{nm}$. At 730 and $850 \mathrm{~nm}$ the probe is sensitive both to vibrational cooling but also to LS state bleaching, thus making the interpretation more difficult. These measurements in the visible and infrared region reveal therefore that even if the system has reached the HS potential within $\approx 200(20) \mathrm{fs}$, more than one picosecond is needed for the HS molecule to thermalize with its environment.
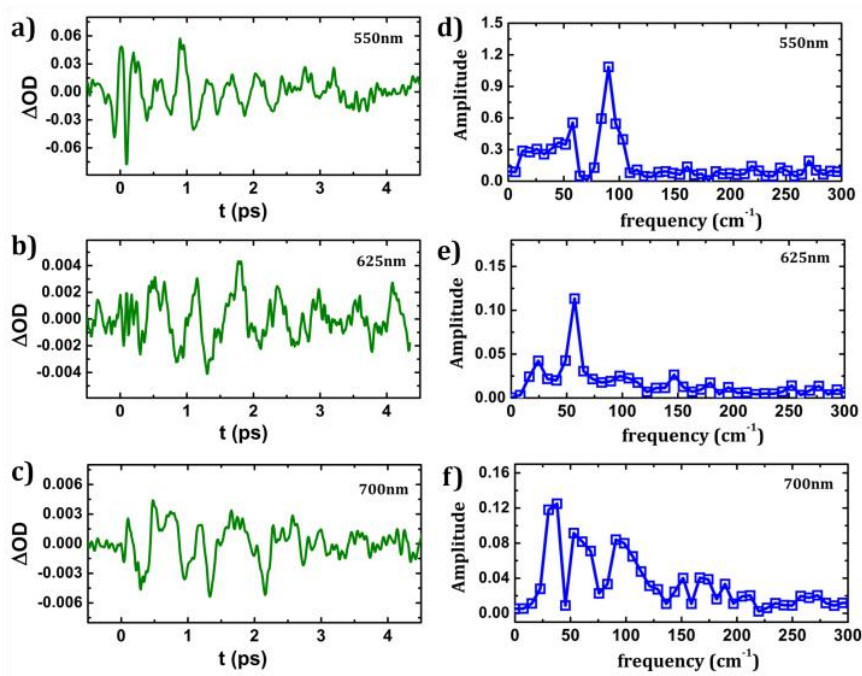

Fig. 6. (a-c), Oscillating component of OD at 550, 625, and $700 \mathrm{~nm}$. (d-f) corresponding FFT of the experimental data, showing the activation of different modes around 85,56 et $35 \mathrm{~cm}^{-1}$.

The $\tau_{v c} \approx 1.6 \mathrm{ps}$ observed here corresponds to the transfer of the excess energy deposited on the molecule by the laser to the lattice. This timescale is shorter than that reported for Fe" molecules in solution (up to 10 ps) with infrared experiments ${ }^{10,11}$. This may be due to the fact that in crystals the excited molecules interact efficiently with the lattice system via phonon-phonon coupling.

\section{Coherent structural oscillations}

A closer inspection of the $\triangle O D$ time traces in Fig. 3-4 allows the observation of weak oscillating components. Fig. 6 (a-c) shows the deviation of the OD data from the exponential fits for different probe wavelengths. The corresponding Fourier transforms of the residual parts corresponding to positive delay are presented in Fig. 6 (d-f). At $550 \mathrm{~nm}$, a prevailing mode is observed around $85 \mathrm{~cm}^{-1}$ (similar oscillations are observed at 525 and $475 \mathrm{~nm}$ in Fig. 3). At the wavelength on the isobestic point $(625 \mathrm{~nm})$ another mode is observed around $56 \mathrm{~cm}^{-1}$. These modes are equally observed at $700 \mathrm{~nm}$, where a third mode around $35 \mathrm{~cm}^{-1}$ also appears.

A similar vibrational coherence in the photoinduced high-spin state was reported for $\mathrm{Fe}^{11} \mathrm{SCO}$ molecules in solution ${ }^{21}$ and more recently in $\left[\mathrm{Fe}(\text { phen })_{2}(\mathrm{NCS})_{2}\right.$ ] crystals $^{14}$. The mechanism behind the high quantum efficiency of LIESST in Fe" SCO compounds was theoretically proposed to result from the instantaneous coupling of the excited (MLCT) state with the phonons of the final (HS) state ${ }^{13}$. The first experimental proof of this process was provided by femtosecond optical pumpprobe spectroscopy studies of LIESST in $\left[\mathrm{Fe}(\text { phen })_{2}(\mathrm{NCS})_{2}\right]$ crystals, where the breathing mode $\left(113 \mathrm{~cm}^{-1}\right)$ associated with the elongation of the Fe- $\mathrm{N}$ bonds was clearly identified ${ }^{14}$ : the instantaneous activation of this molecular breathing phonon drives the system from the MLCT to the HS potential within $170 \mathrm{fs}$, i.e. on the timescale of a half period of such molecular vibration. The fast damping of the key molecular phonon modes leads to the efficient trapping the HS state. It may also result from intermolecular contacts. 

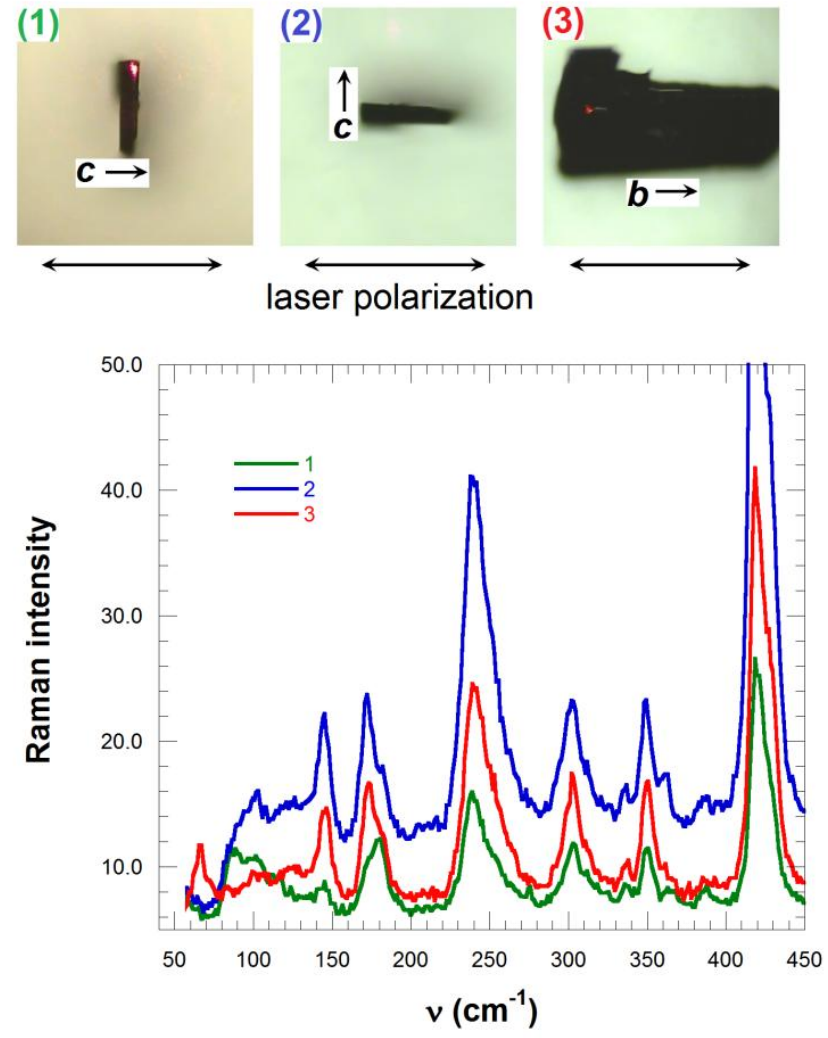

Fig. 7. Raman spectra obtained on single crystals for different polarization (1), (2) and (3) shown in the photographs on the top.

The present observation of the activation of such coherent structural vibrations during LIESST in Fe"I SCO crystals may therefore be also attributed to such mechanism. However, there are very few vibrational spectroscopy studies of Fe $\mathrm{III}^{\prime \prime}$ compounds in literature, even though these low frequencies vibrations are often attributed to ligand breathing or torsion modes $^{22}$. We thus performed Raman measurements at room temperature, as well as calculations of molecular vibrations on the [Fe(3-MeO-SalEen)] $\mathrm{PF}_{6}$ compound for gaining insights on the frequencies and nature of such modes.

\section{Raman and DFT studies of the HS phase}

We used a single crystal for finding the crystal axis orientation with $x$-ray diffraction and checking polarization effect on Raman signal. Fig. 7 shows the single crystal Raman spectra obtained at room temperature. Since the crystalline space group is $P_{\overline{1}}$ and the molecules are in general positions in the unit cell ${ }^{16 a}$, the modes split in two categories: the totally symmetric ones ( $A_{g}$ and Raman active) where molecules oscillate in phase with respect to inversion symmetry and the antisymmetric ones $\left(A_{u}\right.$ and $I R$ active). The Raman spectra reveal several low frequency modes, especially around 65,85 , $100,145,172,181,240$ and $260 \mathrm{~cm}^{-1}$. In this frequency range, the modes may be the skeletal modes of the $\left[\mathrm{FeN}_{4} \mathrm{O}_{2}\right]$ core, but may also correspond to ligand distortions. Because of the $C_{i}$ point symmetry of the crystal, there is no polarization selection: only the Raman intensity may depend on the sample orientation with respect to the laser polarization.

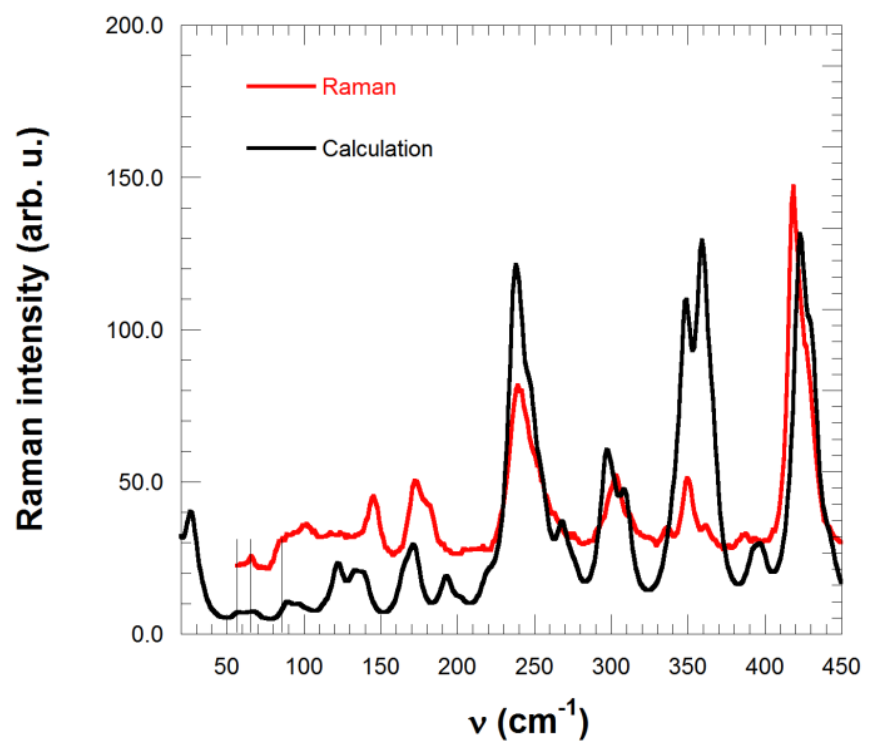

Fig. 8. Comparison of the calculated and experimental (averaged of the different polarizations) Raman spectra.

According to Fig. 7 the relative intensity of the different modes changes with sample orientation. The Raman mode around 85 $\mathrm{cm}^{-1}$ corresponds well to the coherent vibration observed during LIESST in the present Fe" compound. The modes at 35 and $56 \mathrm{~cm}^{-1}$ could not be measured with our Raman set-up because of the dielectric edge filter cut-off.

For understanding better the nature of the molecular vibrations involved during LIESST, we carried out molecular vibration frequencies calculations with DFT methods for an isolated [Fe(3-MeO-SalEen) $\left.{ }_{2}\right] \mathrm{PF}_{6}$ complex, after geometry optimization, by using hybrid B3LYP functional with triple- $\zeta 6$ $311 \mathrm{~g}(\mathrm{~d}, \mathrm{p})$ basis set within Gaussian09 code $^{23,24}$. In Fig. 8 we compare the calculated Raman spectrum with the experimental results from Fig. 7, averaged over the different orientations measured on a single crystal for better comparison with calculations. The overall calculations reproduce quite well the observed Raman spectrum. A complete analysis of the modes and the nature of the vibrations will be published elsewhere and in this paper we focus on the modes observed with time-resolved spectroscopy. In the low frequency region, the calculated modes at 88,56 and $33 \mathrm{~cm}^{-1}$ are close to those observed by Raman and/or time-resolved spectroscopy. The mode at 56 $\mathrm{cm}^{-1}$ (shown in video S1) is mainly associated with $\mathrm{Fe}-\mathrm{N}$ breathing of the $\mathrm{FeN}_{4} \mathrm{O}_{2}$ coordination sphere. The modes at $33 \mathrm{~cm}^{-1}$ (video S2) and $88 \mathrm{~cm}^{-1}$ (video S3) are mainly ligand torsion modes. Since during the LS to HS conversion the structural reorganization involves both the molecular breathing (as Fe-L bonds elongate) and the ligand torsion, such modes should play an important role during the self-trapping process.

For understanding why different vibration modes are observed during LIESST depending on the probe wavelength, we need to analyze optical absorption of HS species through an accurate description of excited states and a time dependent DFT (TDDFT) approach is required ${ }^{25}$. 


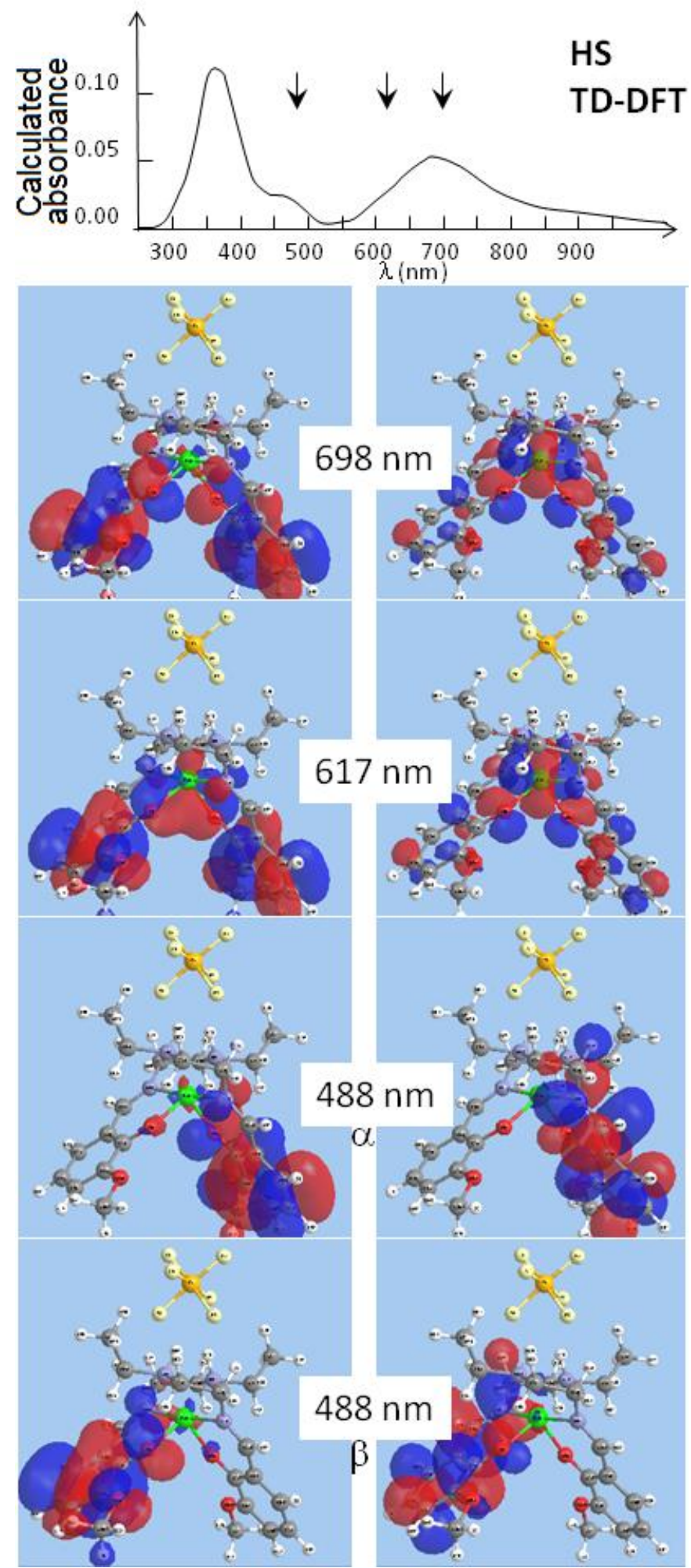

Fig. 9. Natural transition orbitals of hole (left) and particle (right) calculated in the case of $\mathrm{HS}$ species at 698,617 and $488 \mathrm{~nm}$, i.e. close to the experimental probes at 700,625 and $550-475 \mathrm{~nm} . \alpha$ and $\beta$ designate spin $\uparrow$ and spin $\downarrow$. The calculated absorbance spectrum (top) underline the strong weight of these transitions.

The obtained natural transition orbitals (NTO) taking into account the hole-particle pairs resulting from light absorption can then be used to interpret our data.

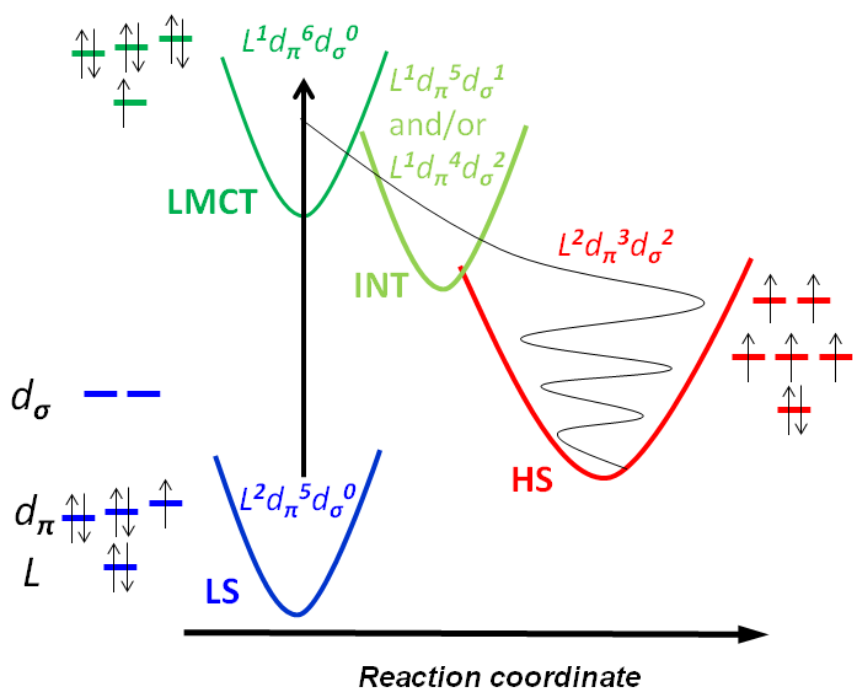

Fig. 10. Schematic representation of the photoswitching trajectory along a simple reaction coordinate.

Fig. 9 shows the NTO corresponding to the hole-particle transition state around 500, 617 and $700 \mathrm{~nm}$. The strong weight of the particle orbital on the Fe- $\mathrm{N}$ (and Fe-O) bonds for the transition at $617 \mathrm{~nm}$ explains the sensitivity to the breathing mode of the pump-probe data at $625 \mathrm{~nm}$. The transition state around 700 or $500 \mathrm{~nm}$ are characterized by a strong weight of the particle orbital mainly located on one ligand and explain the sensitivity to ligand torsion.

\section{Ultrafast LIESST dynamics in Fe"' systems}

Our investigation gives important insights on the ultrafast mechanism of LIESST in a Fe ${ }^{\text {III }}$ system, schematically represented in Fig. 10. Molecular orbital calculations (Fig. 2) indicate that the photo-excitation of the LS $\left(L^{2} d_{\pi}^{5} d_{\sigma}{ }^{0}\right)$ generates a LMCT state $\left(L^{1} d_{\pi}^{6} d_{\sigma}{ }^{0}\right)$. Time-resolved spectroscopy data show that the LMCT state decays to the HS state $\left(L^{2} d_{\pi}{ }^{3} d_{\sigma}{ }^{2}\right)$, possibly through other intermediate electronic states such as $L^{1} d_{\pi}^{5} d_{\sigma}{ }^{1}$ or $L^{1} d_{\pi}{ }^{4} d_{\sigma}{ }^{2}$. However, since the HS potential is reached within $\approx 200 \mathrm{fs}$, these electronic states only serve as mediators and are dynamically mixed. The different photoexcited molecules arrive "simultaneously" on the HS potential, where they oscillate. The global coherent oscillations experimentally observed underline that the phase is mainly kept between the different photo-excited molecules. Indeed, the observation of vibrational coherence in the photoinduced HS state with oscillation around $85 \mathrm{~cm}^{-1}$ (392 fs period), requires that the photoexcited molecules reach the HS potential on a timescale shorter than a half oscillation period to avoid dephasing. This is in agreement with $\tau_{I S C}=200 \pm 20 \mathrm{fs}$ observed by time-resolved spectroscopy (Fig. 4). This very short time-scale is similar to the one reported for LIESST in Fe" SCO systems ${ }^{10,14}$ and also indicates that intermediates other than LMCT and HS states mainly serve as mediators. 
The instantaneous activation of several phonons of the HS state results from the important displacive nature of the mechanism. However, the structural change between LS and HS structures involves several degrees of freedom such as molecular breathing, torsion..., which may be activated with different timescales. Therefore, the transformation pathway connecting the initial photo-excited state to the photoinduced HS state is only roughly described by the classical single coordinate picture employed here in Fig. 10. A more complex multi-dimensional energy surface description has to be used for a more detailed analysis. However, these results indicate that on such short time-scale the wave functions describing the electronic and structural degrees of freedom are strongly coupled during the process. This LS-to-HS photoswitching dynamics is therefore very different in nature from the reverse HS-to-LS one observed recently in a Fe" SCO material, where a true (vibrationally cooled) triplet state is populated and depopulated within 10 's $\mathrm{ps}^{9}$. Indeed, such slow reverse-LIESST dynamics obey the Born-Oppenheimer approximation.

\section{Local nature of ultrafast LIESST}

Another important question associated with LIESST in crystals is the possibility of cooperative response to light excitation. We look into possible cooperativity on ps timescale by following the fraction $\Delta X_{H S}$ of molecules converted after $5 \mathrm{ps}$ from LS to HS state. It is monitored by femtosecond optical spectroscopy for different excitation densities and for different temperature (and therefore different initial fractions of molecules in LS states as shown in Fig. 1). Fig. 11 shows how $\Delta X_{H S}$ changes with the pump laser fluence $F$ (i.e. on the excitation density). It is clear that $\Delta X_{H S}$ depends linearly on $F$, i.e. there is no amplification on such ultra-short time scale. The observed slopes in Fig. 11 correspond to the photo-response and are given here in $\Delta X_{H S} / F$. The slopes measured for different temperatures, proportional to the number of photoswitched molecules per incident photon, change with temperature. This is not surprising because the fraction of molecules in the LS state decreases above $120 \mathrm{~K}$ (Fig. 1). Therefore the fraction of molecules switched from LS to HS states, should be weighted both by $X_{L S}$, the initial fraction of molecules in the LS state at a given temperature $\left(X_{L S}=1-X_{H S}\right)$, and by the laser fluence $F$.

$$
\Delta X_{H S} \propto F \times X_{L S} \text { or } \Delta X_{H S} / F \propto X_{L S}
$$

Fig $11(\mathrm{~b})$ shows that the photo-response $\Delta X_{H S} / F$ depends linearly on $X_{L S}$. Incidentally, based on such plot we can also conclude on the absence of back conversion with the same pump wavelength (from HS to LS), since in such a case the photo-response would be negative as $X_{L S}$ will tend to 0 . These important results underline the local nature of the photoswitching process occurring within a picosecond. Each individual photo-switching process can be regarded as an isolated molecular event. It is only on longer time-scale that macroscopic conversion occur, driven by elastic and thermal mechanisms $^{18}$.
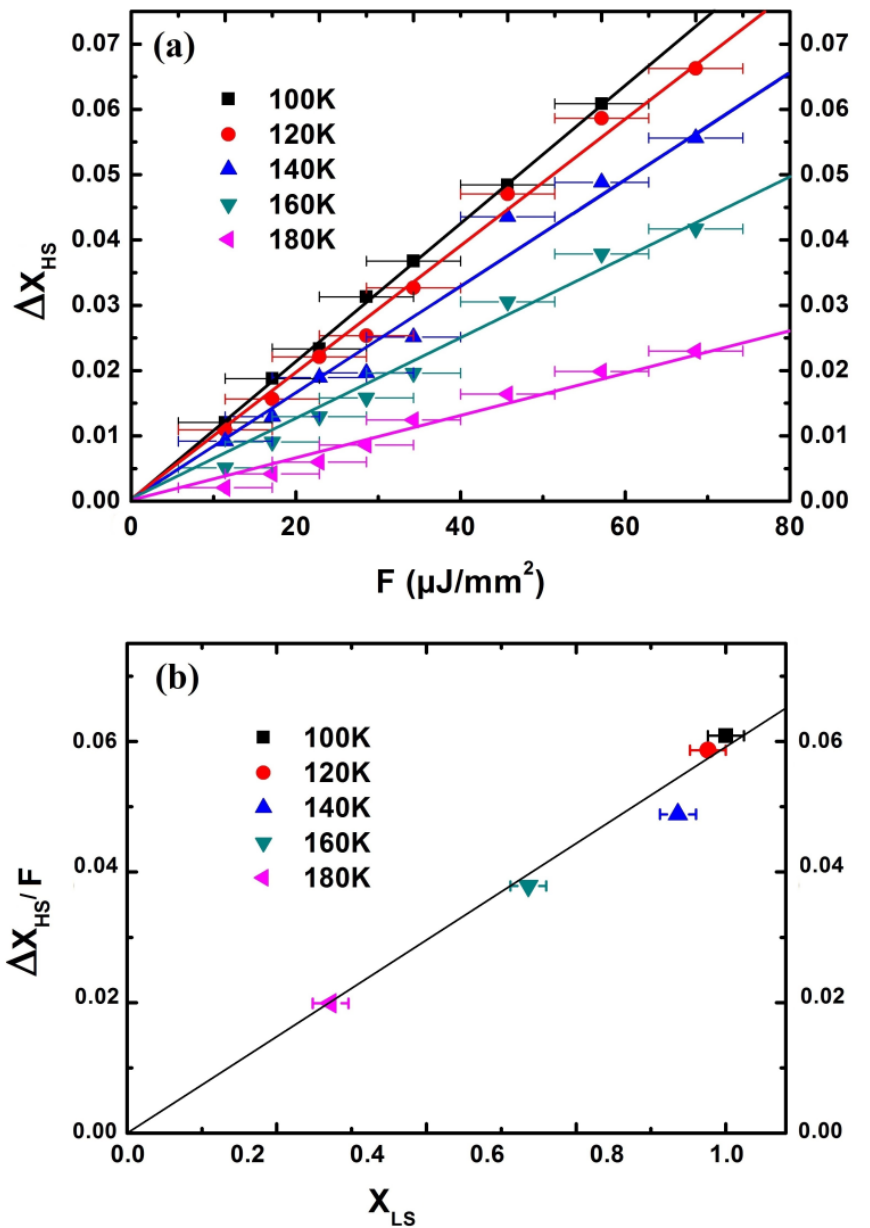

Fig. 11. (a) Temperature dependence of the fraction of molecules photo-switched to the HS state $\left(\Delta X_{H S}\right)$ for different pump fluencies. (b) Dependence of the photo-response $\left(\Delta X_{H S} / F\right)$ with the initial fraction of LS molecules $\left(X_{L S}\right)$.

\section{Conclusions}

This detailed study allows to draw a complete picture of the LIESST photo-switching process in Fe' systems in the solid state. First of all, the photo-response is local on ps timescale, as characterized by the linear dependence with the number of photons and with the initial fraction of molecules in the LS state. This confirms that the initial mechanism might be seen as a local molecular event. Our new data in the present Fe ${ }^{\text {III }}$ system also reveal that during the LIESST process several key modes are coherently activated. They are associated with changes of relevant reaction coordinates accompanying change of spin state (breathing, torsion...). The $\mathrm{Fe}-\mathrm{N}$ lengthening is shorter in $\mathrm{Fe}^{\mathrm{III}}(0.15 \AA)$ than in $\mathrm{Fe}^{\text {II }}(0.20 \AA)$ and the frequency of the breathing modes differ. If a clear breathing mode (in phase elongation of the $6 \mathrm{Fe}-\mathrm{N}$ bonds) is easily identified in Fe" $\mathrm{N}_{6}$ systems (around $113 \mathrm{~cm}^{-1}$ ) ${ }^{14}$ the lower symmetry of the present compound with $\mathrm{Fe}^{\prime \prime \prime} \mathrm{N}_{4} \mathrm{O}_{2}$ coordination sphere makes such identification more difficult and several modes may involve $\mathrm{Fe}-\mathrm{N}$ elongation (as it is the case of the $56 \mathrm{~cm}^{-1}$ mode). In addition, the breathing frequencies depend on the ligand (mass) and on the strength 
of its bonding to the metal. However, our results indicate that the mechanism behind the ultrafast intersystem crossing in $\mathrm{Fe}^{\prime \prime}$ and other transition-metal complexes ${ }^{13,14}$, described in terms of the dephasing of the photoexcited state to the phonon continuum of the photoinduced (here HS) state with significantly different structure, is also valid for describing the photo-switching mechanism in $\mathrm{Fe}^{\mathrm{III}}$ systems. The observed structural coherence underlines the dynamical nature of the light-induced excited spin-state trapping process, as opposed to the so far prevailing kinetic description based on simple exponential decay from photo-excited to photoinduced states.

\section{Experimental}

\section{Sample preparation}

The molecular compound [Fe(3-MeO-SalEen $\left.)_{2}\right] \mathrm{PF}_{6}$ was synthesized in the form of a microcrystalline powder and single-crystals as previously described ${ }^{16 a, 18}$. Nanocrystals ${ }^{16 c}$ were prepared by rapidly adding a concentrated solution of salt $(10 \mathrm{mg})$ in acetone $(1 \mathrm{~mL})$ into a large volume $(20 \mathrm{~mL})$ of butan-1-ol maintained at $-40{ }^{\circ} \mathrm{C}$ under a vigorous stirring. After 15 minutes, the particles were isolated by centrifugation, rinsed with pentane, dried. The properties of needle-shaped nanocrystals (typical dimensions of 900(230)x285(65)×64(18) $\mathrm{nm}^{3}$ from TEM) were checked with powder X-ray diffraction, IR and magnetism ${ }^{16 c}$. The nanocrystals were processed in PVP polymeric films. $5 \mathrm{mg}$ of nanocrystals were added to a viscous solution of $100 \mathrm{mg}$ of PVP (MM $\left.=45000 \mathrm{gmol}^{-1}\right)$ in $500 \mu \mathrm{L}$ of butan-1-ol; the mixture was sonicated (30 $\mathrm{min})$, then spincoated on glass substrates (1000 rpm) and dried at air before use.

\section{Femtosecond optical spectroscopy}

For tracking the photo-induced spin state switching dynamics in real time, we employ femtosecond pump probe transmission measurements ${ }^{17 a}$. The laser beam was provided from the output of a femtosecond regenerative amplifier (Legend Elite, Coherent) and then split in two beams, each of them seeding an optical parametric amplifier (TOPAS, Light Conversion) allowing to cover spectral range from UV to infrared. The experimental time resolution is $140 \mathrm{fs}$. The pump wavelength was set at $850 \mathrm{~nm}$ corresponding to the LMCT absorption band. The photo-excited HS state relaxes towards the LS state in less than $1 \mathrm{~ms}$, allowing the $1 \mathrm{kHz}$ repetition rate of the experiment. The sample was cooled down using a nitrogen cryostream. The one colour probe beam, tuned across a broad spectral range (480-950 nm) measured transient optical density. We could measure the variation of the fraction of HS molecules $\Delta X_{H S}(\mathrm{t})$ by scaling $\triangle O D(\mathrm{t})$, the change of OD in time between LS and photo-excited HS state, to the OD change between LS and HS states at thermal equilibrium.

\section{Raman spectroscopy}

Raman spectra were collected in the $55-1200 \mathrm{~cm}^{-1}$ low frequency range using a LabRAM-HR Raman spectrometer (Horiba / Jobin Yvon). A $100 \times 300 \times 30 \mu \mathrm{m}^{3}$ single crystal has been studied for different polarisation configurations. The $632.818 \mathrm{~nm}$ line of a $15 \mathrm{~mW}$ He-Ne laser was used as the excitation source. The exciting radiation was directed through a neutral density filter (optical density 2) to avoid sample heating problems and was focused on the sample via a $\times 100$ long working-distance objective. The scattered light was collected in backscattering configuration and the Rayleigh scattering was removed by means of a dielectric edge filter.

\section{DFT calculations}

Molecular vibration frequencies calculations were carried out for [Fe(3-MeO-SalEen)] $\mathrm{PF}_{6}$ after geometry optimization, by using hybrid B3LYP functional with triple- $\zeta 6-311 \mathrm{~g}(\mathrm{~d}, \mathrm{p})$ basis set within Gaussian09 code $^{23}$. Frequencies are determined from the second derivatives of the energy with respect to the atomic positions and then operating transformation to massweighted coordinates. Exploring the results especially for the vibrations and their animations with screen captures was done with Gaussview annex module to Gaussian.

TD-DFT as implemented in the Gaussian 09 package ${ }^{23}$ was applied for obtaining the NTO of the HS [Fe(3-MeO-SalEen)] $\mathrm{PF}_{6}$ system, starting from preliminary geometry optimized molecule with B3LYP/6-311g $(d, p)$ functional-basis set.

\section{Acknowledgements}

This work was supported by the Institut Universitaire de France, Rennes Métropole, Région Bretagne (CREATE 4146), ANR (ANR-13-BS04-0002) and Fonds Européen de Développement Régional (FEDER). Raman measurements have been performed on the SIR raman system from the ScanMAT platform of Rennes 1 University.

\section{Notes and references}

1 a) D. Polli, P. Altoe, O. Weingart, K.M. Spillane, C. Manzoni, D. Brida, G. Tomasello, G. Orlandi, P. Kukura, R.A. Mathies, M. Garavelli, and G. Cerullo, Nature, 2010, 467, 440-443; b) A. H. Zewail. Angew. Chem. Int. Ed. 2000, 39, 2586-2631; c) J.H Lee, M. Wulff, S. Bratos, J. Petersen, L. Guérin, J.C Leiknman, M. Cammarata, Q.Y. Kong, J. Kim, K. Moller, and H.J. Hee, J. Am. Chem. Soc., 2013, 135, 3255.

2 a) M. Gao, C. Lu,H. Jean-Ruel, L. C. Liu, A. Marx, K. Onda, S. Koshihara, Y. Nakano, X. Shao, T. Hiramatsu, G. Saito, H. Yamochi, R. R. Cooney, G. Moriena, G. Sciaini, and R.J.D. Miller, Nature, 2013, 496, 343-346; b) T. Ishikawa, N. Fukazawa, Y. Matsubara, R. Nakajima, K. Onda, Y. Okimoto, S. Koshihara, M. Lorenc, E. Collet, M. Tamura and R. Kato, Physical Review B, 2009, 80, 115108; c) Y. Kawakami, S. Iwai, T. Fukatsu, M. Miura, N. Yoneyama, T. Sasaki, and N. Kobayashi, Phys. Rev. Lett., 2009, 103, 066403.

3 a) H. Okamoto, Y. Ishige, S. Tanaka, H. Kishida, S. Iwai, and Y. Tokura, Phys. Rev. B, 2004, 70, 165202; b) L. Guérin, J. Hébert, M. Buron-Le Cointe, S. Adachi, S. Koshihara, H. Cailleau, and E. Collet, Phys. Rev. Lett., 2010, 105, 246101.

4 M. Halcrow, Ed., Spin-crossover materials (Wiley, West Sussex, 2013) ISBN 9781119998679.

5 a) S. Decurtins, P. Gütlich, C.P. Köhler, H. Spiering, and A. Hauser, Chem. Phys. Lett., 1984, 105, 1; b) A. Hauser, Chem. Phys. Lett., 1992, 192, 65-70.

6 J.F. Létard, L. Capes, G. Chastanet, N. Moliner, S. Létard, J.A. Real, and O. Kahn, Chem. Phys. Lett., 1999, 313, 115. 
7 a) N. Bréfuel, H. Watanabe, L. Toupet, J. Come, N. Matsumoto, E. Collet, K. Tanaka and J.-P. Tuchagues, Ang. Chem. Int. Ed., 2009, 48, 304-9307; b) E. Collet, H. Watanabe, N. Bréfuel, L. Palatinus, L. Roudaut, L. Toupet, K. Tanaka, J.-P. Tuchagues, P. Fertey, S. Ravy, B. Toudic, and H. Cailleau, Phys. Rev. Lett., 2012, 109, 257206.

8 a) R. Bertoni, M. Lorenc, A. Tissot, M. Servol, M.-L. Boillot, and E. Collet, Angew. Chem. Int. Ed., 2012, 51, 7485-7489; b) W. Kaszub, A. Marino, M. Lorenc, E. Collet, E.G. Bagryanskaya, E.V. Tretyakov, V.I. Ovcharenko and M.V. Fedin, Ang. Chem. Int. Ed., 2014, 53, 10636-10640; c) A Marino, M. Buron-Le Cointe, M. Lorenc, L. Toupet, R. Henning, A.D. DiChiara, K. Moffat, N. Bréfuel, E. Collet, Faraday Discussion, 2015, 177, 363-379.

9 A. Marino, P. Chakraborty, M. Servol, M. Lorenc, E. Collet and A. Hauser, Ang. Chem. Int. Ed., 2014, 53, 3863-3867.

10 a) W.Gawelda, A. Cannizzo, V.T. Pham, F. van Mourik, C. Bressler, and M. Chergui, J. Am. Chem. Soc., 2007, 129, 8199-8206; b) A. Canizzo , C. Milne, C. Consani, W. Gawelda, C. Bressler, F. van Mourik, and M. Chergui, Coord. Chem. Rev., 2010, 254, 2677-2686; c) J.K. McCusker, K.N. Walde, R.C Dunn, J.D Simon, D. Madge, and D.N. Hendrickson, J. Am. Chem. Soc., 1992, 114, 6919-6921; d) J.E. Monat, and J.K. McCusker, J. Am. Chem. Soc., 2000, 122, 4092-4097; e) A.L. Smeigh, M. Creelman, R.A. Mathies, and J.K. McCusker, J. Am. Chem. Soc., 2008, 130, 14105-14107; f) M. M. N. Wolf, R. Groß, C. Schumann, J. A. Wolny, V. Schünemann, A. Døssing, H. Paulsen, J. J. McGarvey, and R. Diller, Phys. Chem. Chem. Phys., 2008, 10, 4264-4273; g) I. Lawthers, and J. J. McGarvey, J. Am. Chem. Soc., 1984, 15, 106.

11 a) C. Bressler, C. Milne, V.-T. Pham, A. El Nahhas, R. M. van der Veen, W. Gawelda, S. Johnson, P. Beaud, D. Grolimund, M. Kaiser, C. N. Borca, G. Ingold, R. Abela and M. Chergui, Science, 2009, 323, 489; b) N. Huse, H. Cho, K. Hong, L. Jamula, F.M.F. de Groot, T.K. Kim, J.K. McCusker, and R.W. Schoenlein, J. Phys. Chem. Lett., 2011, 2, 880-884; c) M. Khalil, M. M. Marcus, A. L. Smeigh, J. K. McCusker, H. H. W. Chong and R. W. Schoenlein, J. Phys. Chem. A., 2006, 110, 38; d) W. Gawelda, V.-T. Pham, M. Benfatto, Y. Zaushitsyn, M. Kaiser, D. Grolimund, S. L. Johnson, R. Abela, A. Hauser, C. Bressler and M. Chergui, Phys. Rev. Lett., 2007, 98, 057401; e) S. Nozawa, T. Sato, M. Chollet, K. Ichiyanagi, A. Tomita, H. Fujii, S.-I. Adachi and S.-Y. Koshihara, J. Am. Chem. Soc., 2010, 132, 61; f) H.T. Lemke, C. Bressler, L.X Chen, D.M. Fritz, K.J. Gaffney, A. Galler, W. Gawelda, K. Haldrup, R.W. Hartsock, H. Ihee, J. Kim, K.H. Kim, J.H Lee, M.M. Nielsen, A.B. Stickrath, W. Zhang, D. Zhu, and M. Cammarata, J. Phys. Chem. A, 2013, 117, 735-740.

12 W. Zhang, R. Alonso-Mori, U. Bergmann, C. Bressler, M. Chollet, A. Galler, W. Gawelda, R. G. Hadt, R. W. Hartsock, T. Kroll, K. S. Kjær, K. Kubiček, H. T. Lemke, H. W. Liang, D. A. Meyer, M. M. Nielsen, C. Purser, J. S. Robinson, E. I. Solomon, Z. Sun, D. Sokaras, T. B. van Driel, G. Vankó, T.-C. Weng, D. Zhu and K. J. Gaffney Nature, 2014, 509,345-348.

13 a) M. Van Veenendaal, J. Chang, and A.J. Fedro, Phys. Rev. Lett., 2010 104, 067401; b) J. Chuang, A.J. Fedro, and M. Van Veenendaal, Phys. Rev. B, 2010, 82, 075124.

14 a) M. Cammarata, R. Bertoni, M. Lorenc, H. Cailleau, S. Di Matteo, C. Mauriac, S. F. Matar, H. Lemke, M. Chollet, S Ravy, C. Laulhé, J.-F. Létard and E. Collet, Phys. Rev. Lett., 2014, 113, 227402; b) R. Bertoni, M. Cammarata, M. Lorenc, S. F. Matar, J.-F. Létard, H. T. Lemke and E. Collet Acc. Chem. Res., 2014, DOI: 10.1021/ar500444d

15 a) S. Hayami, Z. Gu, M. Shiro, Y. Einaga, A. Fujishima and O. Sato, J. Am. Chem. Soc. 2000, 122, $7126-7127$; b) S. Hayami, K. Hiki, T. Kawahara, Y. Maeda, D. Urakami, K. Inoue, M. Ohama, S. Kawata and O. Sato, Chem. Eur. J., 2009, 15 3497-3508; c) T. Shimizu, Y. Komatsu, H. Kamihata, Y. H. Lee,
A. Fuyuhiro, S. lijima and S. Hayami, J. Inclusion Phenom. Macrocyclic. Chem., 2011, 71, 363-369; d) G. Juhasz, S. Hayami, O. Sato and Y. Maeda, Chem. Phys. Lett., 2002, 364, 164-170; e) H. Ando, Y. Nakao, H. Sato and S. Sakaki, J. Phys. Chem. A, 2007, 111, $5515-5522$; f) K. D. Murnaghan, C. Carbonera, L. Toupet, M. Griffin, M. M. Dîrtu, C. Desplanches, Y. Garcia, E. Collet, J.F. Létard and G. G. Morgan, Chem. Eur. J., 2014, 20, 5613-5618.

16 a) A. Tissot, R. Bertoni, E. Collet, L. Toupet, and M.-L. Boillot, J. Mat. Chem., 2011, 21, 18347-18353; b) R. Bertoni, M. Lorenc, A. Tissot, M.-L. Boillot and E. Collet, Coord. Chem. Rev., 2015, 282-283, 66-76; c) A. Tissot, L. Rechignat, A. Bousseksou, and M.-L. Boillot, J. Mater. Chem. 2012, 22 3411-3419.

17 a) M. Lorenc, C. Balde, W. Kaszub, A. Tissot, Moisan, M. Servol, M. Buron-Le Cointe, H. Cailleau, P. Chasle, P. Czarnecki, M. L. Boillot and E. Collet, Phys. Rev. B., 2012, 85 054302; b) H. Cailleau, M. Lorenc, L. Guérin, M. Servol, E. Collet and M. Buron-Le Cointe, Acta Cryst. A, 2010, 66, 189; c) E. Collet, M. Lorenc, M. Cammarata, L. Guérin, M. Servol, A. Tissot, M.L. Boillot, H. Cailleau and M. Buron, Chem. Eur. J., 2012, 18, 2051 d) E. Collet, N. Moisan, C. Baldé, R. Bertoni, E. Trzop, C. Laulhé, M. Lorenc, M. Servol, H. Cailleau, A. Tissot, M.L. Boillot, T. Graber, R. Henning, P. Coppens and M. Buron, Phys. Chem. Chem. Phys., 2012, 146192.

18 M.S. Haddad, M.W. Lunch, W.D. Federer, and D.N. Hendrickson, Inorg.Chem., 1981, 20, 123-131.

19 M. Sorai, R. Burriel, E. F. Westrum, and D.N. Hendrickson, J.Phys. Chem. B, 2008, 112, 4344.

20 a) A. Tissot, C. Enachescu and M.-L. Boillot, J. Mater. Chem., 2012, 22, 20451-20457; b) R. Tanasa, J. Laisney, A. Stancu, M.-L. Boillot and C. Enachescu, Appl. Phys. Lett., 2014, 104, 031909.

21 C. Consani, M. Prémont-Schwarz, A. Elnahhas, C. Bressler, F. van Mourik, A. Cannizzo and M. Chergui, Angew. Chem. 2009, 121, 7320.

22 M. Sorai, R. Burriel, E. F. Westrum, and D.N. Hendrickson, J. Phys. Chem. B, 2008, 112, 4344.

23 M. J. Frisch et al, GAUSSIANO3 (revision C.02), Gaussian, Inc. Wallingford CT, 2004.

24 M. P. Andersson and P. Uvdal, J. Phys. Chem. A, 2005, 109, 2937-2941

25 R. Martin, J. Chem. Phys. 2003, 118, 4775 